\title{
A STUDY ON THE ROLE OF ZWITTERIONIC PHOSPHATIDYLCHOLINE LIPID (S) IN PHYSICOCHEMICAL FEATURES OF LIPOSOME ENCAPSULATED PACLITAXEL AS A DRUG DELIVERY SYSTEM
}

\author{
Abdulsalam M. Kassem ${ }^{1,2^{*}}$, Hammam A. A. Mowafy ${ }^{2}$, Sherif K. Abu-Elyazid², Ahmed \\ M. Samy ${ }^{2}$, Keiichiro Okuhira ${ }^{1}$ \\ ${ }^{1}$ Department of Molecular Physical Pharmaceutics, Institute of Biomedical Sciences, \\ Tokushima University, 1-78-1 Sho-machi, Tokushima 770-8505, Japan. \\ ${ }^{2}$ Department of Pharmaceutics and Pharmaceutical Industry, College of Pharmacy \\ (Boys), Al-Azhar University, 1 El-Mokhayam El-Daem St., Nasr City, P.O. Box 11884, \\ Cairo, Egypt.
}

*Corresponding author: dr.abdokasem2011@azhar.edu.eg

\begin{abstract}
The lipid composition of the liposomal membrane plays a crucial role in the physical and pharmacological behaviors of liposomal payloads, and it can be tailored to achieve the desired delivery needs. Thus, the aim of the current study was to develop and optimize paclitaxel (PTX) loaded liposome nanocarrier using a thin-film rehydrating method and studying the influence of different zwitterionic Phosphatidylcholine (PC) lipids with different acyl chains, on the physicochemical properties of the developed PTX liposomal formulations. The studied PC were dipalmitoyl (DPPC), dimyristoyl (DMPC) and palmitoyl-oleoyl (POPC) PCs, in combination with cholesterol. Several characteristics were monitored, including PTX entrapment efficiency (EE) of liposomes, particle size (PS), polydispersity index (PDI), zeta potential (ZP), PTX release in phosphate buffered saline (PBS) with 0.1 tween ${ }^{\circledR} 80$ and in PBS / $10 \%$ fetal bovine serum (FBS) cell culture medium, as well as in vitro antitumoral and therapeutic effects. The results demonstrated that DPPC exhibited superiority in the physicochemical properties when compared with DMPC and POPC. The in vitro cytotoxicity findings indicate that the PTX-liposome would be a promising substitutional approach for Taxol ${ }^{\circledR}$ as a commercial product with a toxic solvent. The results outcomes indicated that the developed PTX-liposome would be a viable drug delivery system with sustaining effect for management of colorectal cancer cells and further clinical use.
\end{abstract}

Keywords :Liposome, Paclitaxel, Cholesterol, zwitterionic Phosphatidylcholine, sustained release. 


\section{Introduction}

Paclitaxel is one of the most effective drugs used in chemotherapy of cancer and is a natural product isolated from bark of the Pacific Yew tree, Taxus brevifolia (Kim and Park, 2017). Its mechanism of action involves interference in the normal breakdown of microtubules during cell division, thereby inducing apoptosis and mitotic arrest, as well as affecting fundamental cellular functions including cell motility, cell transport, and mitosis. PTX is a commonly used anticancer drug that possesses pharmacological activity when applied as a single therapeutic agent and is extensively used to treat ovarian, pancreatic, breast, non-small-cell lung, and other cancers (Huang et al., 2018).

However, due to its hydrophobicity, PTX has poor water solubility $(1 \mu \mathrm{g} / \mathrm{mL})$, which limits its clinical application. The clinical preparation of PTX marketed as Taxol $^{\circledR}$ is composed of Cremophor ${ }^{\circledR}$ EL (polyoxyethylated castor oil) and ethanol (1:1, $\mathrm{v} / \mathrm{v}$ ) and is diluted 10- to 20-fold in $5 \%$ dextrose solution before administration (Kim and Park, 2017). Various advanced drug delivery system based on nanotechnology were developed to face these hardships including, SNEDDS (Ding et al., 2019), nanostructured lipid carrier (Nordin rt al., 2019), silica nanoparticles (Luo et al., 2019) and others.

Liposomes are artificial vesicles that have been extensively applied as a type of drug delivery vehicle to enhance the efficacy of therapy (Davis et al., 2008). Enormous efforts have been made to design liposomal formulations with particular physical, biological, and pharmaceutical features, with the goal of optimizing the therapeutic response. An understanding of the liposome parameters influencing a formulation's therapeutic response are a prerequisite for rational modification of liposomal formulations. These parameters include the liposomes' surface charge, size distribution, physical stability, drug entrapment efficiency, and drug release and cell uptake kinetics (Farzaneh et al., 2018).

The phospholipid composition of liposomal membrane influences the drug loading efficiency and drug release kinetics (Alves et al., 2017). The hydrophobicity, thickness and compactness of the membrane are determining factors in the drug loading efficiency and the leakage of molecules entrapped in the liposomes (Alves et al., 2017).

In the current study, we used different lipid components with defined acyl chains as follows: (a) dipalmitoyl PC (DPPC or di16:0 PC), with a phase transition temperature $\left(\mathrm{T}_{\mathrm{m}}\right)$ of $\sim 41{ }^{\circ} \mathrm{C}$; (b) dimyristoyl PC (DMPC or di14:0 PC), with a $\mathrm{T}_{\mathrm{m}}$ of $\sim 23{ }^{\circ} \mathrm{C}$; and (c) palmitoyl-oleoyl PC (POPC; typically (C16:0-18:1 PC), with a $\mathrm{T}_{\mathrm{m}}$ below $0{ }^{\circ} \mathrm{C}$ (OhvoRekilä et al., 1998). Several aspects of these liposomes have been examined, including liposome PS, size distribution, as well as PTX EE, PTX release in different media, liposomal cell uptake and cytotoxicity. 


\section{Experimental}

\section{Materials}

Paclitaxel (purity > 99.5 \%) was acquired from LC Laboratories, Woburn, MA., (USA). Taxol ${ }^{\circledR}$ was supplied by Bristol-Myers Squibb KK, Tokyo, (Japan). 1,2dipalmitoyl-sn-glycero-3-phosphocholine (DPPC; COATSOME MC-6060), 1,2Dimyristoyl-sn-glycero-3-phosphocholine (DMPC; COATSOME MC-4040) and1palmitoyl-2-oleoyl-sn-glycero-3-phosphocholine (POPC; COATSOME MC-6081) were provided by Nippon Oil and Fats Co., Ltd, (Japan). Cholesterol was provided by Sigma Aldrich, Inc., St., Louis, MO., (USA). 2 [4-(2-hydroxyethyl)-1-piprazenyl] ethanesulfonic acid (HEPES) and Hoechst 33342 stain were acquired from Dojindo Lab., Kumamoto, (Japan). Highly purified milli-Q water (resistivity of $18.2 \mathrm{MX} \mathrm{cm}$ ). Acetonitrile (HPLC grade) was supplied by Wako Pure Chemical, Osaka, (Japan).

\section{HPLC analysis of PTX}

Determination of PTX was performed according to a previously reported method with minor modifications (Rui et al., 2017). The high performance liquid chromatography (HPLC) system used was Shimadzu corporation, Kyoto, (Japan), equipped with a UV / VIS detector (Shimadzu SPD-20A / 20AV, Japan), a pump (Shimadzu LC-20AT, Japan), degaser (Shimadzu DGU-20A3, Japan), column oven (Shimadzu CTO-20A, Japan) and an automated sampling system (Shimadzu SIL-20A / 20AC Autosampler, Japan).

The mobile phase (acetonitrile / water, 3:1, v / v) was flowed over a reversedphase $\mathrm{C} 18$ column $\left(\mu\right.$-Bondapak ${ }^{\mathrm{TM}}, 4.6 \times 150 \mathrm{~mm}, 5 \mu \mathrm{m}$ particle size). The effluent was monitored at a flow rate of $0.7 \mathrm{~mL} / \mathrm{min}$ at room temperature. Aliquot $(20 \mu \mathrm{L})$ of the samples was injected and the drug was quantified by its UV absorbance at $\lambda_{\max } 227 \mathrm{~nm}$ and using a calibration curve $\left(\mathrm{R}^{2}\right.$ : 0.9999). Data acquisition and integration were processed with the Lab Solutions LC / GC software version 5.82 (Shimadzu corporation, Kyoto, Japan, 2015).

\section{Preparation of Liposomes}

Liposome nanoparticles were prepared by thin film rehydrating method as reported previously with some modifications (Kim et al., 2019). At first, $15 \mathrm{mg}$ of zwitterionic phosphatidylcholine (PC) lipids, $7.5 \mathrm{mg}$ cholesterol and $1.5 \mathrm{mg}$ PTX were dissolved in chloroform. The mixture was dried under nitrogen flow for $2 \mathrm{~h}$ and then in a vacuum oven overnight at room temperature. The lipid film was rehydrated with

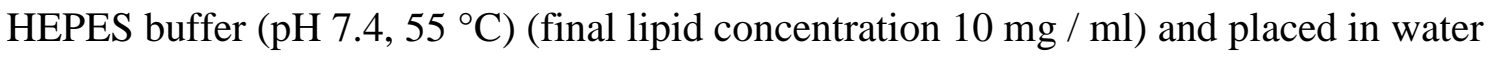
bath at $55{ }^{\circ} \mathrm{C}$ for 1.5 hours with vortex every 15 minutes, for easier formation of (multilamellar vesicles) MLVs. 
Small unilamellar vesicles (SUVs) were formed from MLVs (obtained in the thin film method), using the additional step of sonication. The suspension was sonicated for $30 \mathrm{~min}$ with $10 \mathrm{~s}$ on-off cycles using a probe sonicator (Branson Ultrasonicator, SLPe 40:0.15, Mexico). The mixtures were centrifuged (Eppendorf Centrifuge 5415R, Eppendorf AG 22331 Hamburg, Germany) at $15000 \mathrm{~g}$ for $15 \mathrm{~min}$ for purification of nanoparticles. The supernatant was stored at $-4{ }^{\circ} \mathrm{C}$ until used. Finally, the effect of lipid: drug ratio on encapsulation efficiency was investigated using different ratios of 1:5, 1:10, 1:15, 1:30 (w/w). The experiment was repeated in triplicate.

\section{Characterization of liposomes nanoparticles}

\section{Physicochemical properties of Liposomes}

The average PS, ZP, and PDI of the prepared nanoparticles were determined by Malvern ${ }^{\circledR}$ Zetasizer Nano Zs 90 Malvern ${ }^{\circledR}$ Instruments Limited, Worcestershire, (UK). Each sample was diluted with HEPES buffer (1:100), filtered through $0.22 \mu \mathrm{m}$ filter and gently agitated prior to testing using a $90^{\circ}$ scattering angle at $25^{\circ} \mathrm{C}$.

\section{Entrapment efficiency (EE \%)}

For studying the encapsulation efficiency, liposome nanoparticles were dissolved in acetonitrile for breaking the nanodisc and dissolving PTX. Then, PTX concentration was determined by the reverse-phase HPLC at $227 \mathrm{~nm}$. The encapsulation efficiency was calculated as per the following:

$$
\mathrm{EE}(\%)=\frac{\text { Encapsulated amount of PTX in Liposomes }}{\text { Ttal amount added }} x 100
$$

\section{Transmission electron microscopy}

The morphology of liposome loaded PTX was observed using transmission electron microscopy (TEM; JEOL 1200 EX II, JEOL Ltd., Tokyo, Japan), operating at $80 \mathrm{kV}$ and 60.000-fold magnification. Prior to analysis, PTX-liposomes has diluted appropriately and deposited onto a carbon-coated copper grid followed by staining with $2 \%(\mathrm{w} / \mathrm{v})$ sodium phosphotungstate solution and drying at room temperature.

\section{In vitro dissolution studies}

The in vitro release profile test was performed for the developed PTX-liposome and $\mathrm{Taxol}^{\circledR}$ in $10 \%$ FBS cell culture medium as a biomimetic condition or PBS (pH 7.4) using the dialysis method (Rui et al., 2017). Accurately, an aliquots equivalent to $0.5 \mathrm{mg} / \mathrm{mL}$ from each sample was placed in (spectra / pro ${ }^{\circledR}$ dialyzing, Float-A-Lyzer ${ }^{\circledR}$ G2, with Biotech Cellulose Ester Membrane of molecular weight cut off 10,000-12,000 Da, USA), tightly sealed at both end and soaked in $500 \mathrm{ml}$ of dissolution media 
containing $0.1 \%$ Polysorbate 80 at $37 \pm 0.5^{\circ} \mathrm{C}$ and $100 \mathrm{rpm}$. At the predesigned time intervals, one $\mathrm{ml}$ was withdrawn and replenished with the same volume of fresh medium and eventually, the samples were quantified by the validated HPLC method.

\section{Stability against lipoprotein adsorption}

To investigate the stability of PTX-liposome nanoparticles under biological conditions, the particle diameters and particle size distribution were measured overtimes. Accurately, nanoparticles $(0.5 \mathrm{mg} / \mathrm{ml})$ were mixed with $10 \%$ FBS culture medium $(1: 1 ; \mathrm{v} / \mathrm{v})$ and incubated at $37{ }^{\circ} \mathrm{C}$. At the predetermined time interval $(0,1,2$, and $3 \mathrm{~h}$ ), the samples were diluted with HEPES buffer and assessed by Dynamic Light Scattering method (DLS).

\section{Cell culture}

The Colon 26 (C26) was purchased from the Cell Resource Center for Biomedical Research (RIKEN RBC CELL BANK, Saitama, Japan) was cultured in Dulbecco's Modified Eagle Medium (DMED) culture medium-high glucose level (Wako Pure Chemical, Osaka, Japan). The cells were incubated at $37^{\circ} \mathrm{C}$ in a humidified atmosphere with $5 \% \mathrm{CO}_{2}$. The culture media was supplemented with $10 \% \mathrm{FBS}$, $100 \mu \mathrm{g} / \mathrm{ml}$ streptomycin and $100 \mathrm{IU} / \mathrm{ml}$ penicillin (MP Biomedicals, CA, USA). All experiments were conducted in the logarithmic phase of growth.

\section{In vitro anti-tumoral activity}

The C26 cells were seeded in 96-well microtiter plate at the density of $5 \times 10^{3}$ viable cells per well and incubated $24 \mathrm{~h}$ to allow good adherence. Besides, the cells incubated with taxol ${ }^{\circledR}$, PTX encapsulated liposomes at a concentration of $(0,20,50$, 100, 250, 500 and $1000 \mathrm{~nm}$ ). Additionally, the cells were treated with liposome free drug at the comparable concentration for 72 hours at $37{ }^{\circ} \mathrm{C}$.

After 3 days, $10 \mu \mathrm{l}$ of cell counting kit-8 (CCK-8) (Dojindo Lab., Kumamoto, Japan) was added to each well and incubated at $37{ }^{\circ} \mathrm{C}$ for 3 hours. Finally, the absorbance was measured at $450 \mathrm{~nm}$ using multimode plate reader (Perkin Elmer, Waltham, MA, USA). The cells without the addition of CCK-8 were used as blank to calibrate the spectrophotometer to zero absorbance. The untreated cells were taken as control with $100 \%$ viability. 


\section{Results and Discussion}

\section{HPLC assay of PTX}

Figure $(1, \mathrm{~A})$ showed the chromatogram of PTX in mobile phase. It is obvious from the chromatogram that well-resolved peak was obtained. The retention times of PTX in mobile phase was 7.94 min. The standard curve of PTX by HPLC method was constructed at $227 \mathrm{~nm}$. An excellent linearity was obtained for PTX of concentration in range of $0.06-1000 \mu \mathrm{g} / \mathrm{ml}$ with a good determination coefficient $=1$. The equation obtained of the standard curve was $y=2943.2 x-3639$. The above data was represented graphically in figure $(1, \mathrm{~B})$.
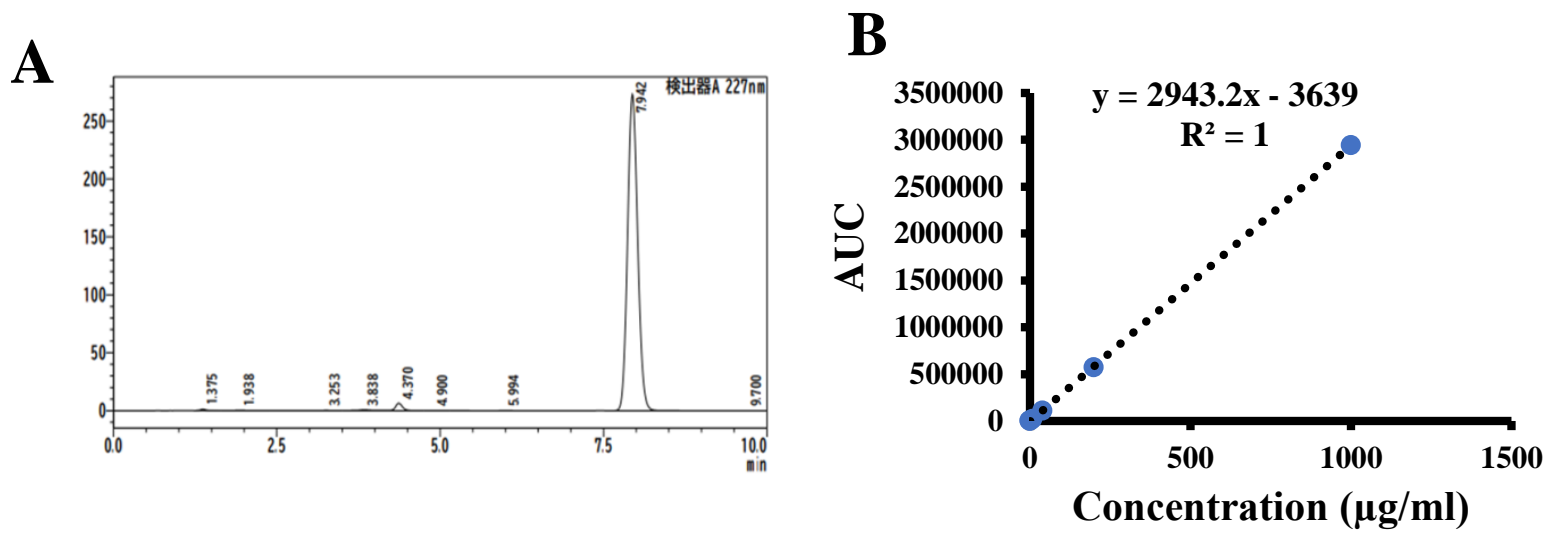

Figure (1): HPLC analysis of PTX using acetonitrile / water (3:1, v / v) as mobile phase. (A) The HPLC chromatogram of PTX only in mobile phase at $1000 \mu \mathrm{g} / \mathrm{ml}$. (B) The standard curve of PTX at $227 \mathrm{~nm}$ by RP-HPLC method

\section{Effect of phosphatidylcholines on physicochemical characteristics of liposomes loaded PTX}

For the initial lipid and peptide screening, the lipid: cholesterol: PTX was fixed at 1: 0.5: $0.1 \mathrm{w} / \mathrm{w}$, respectively. As seen in table (1), Different optimization parameters were used for selection the optimized formulation. Different types of phospholipids affect the formation and stability of liposomes. Thus, it is necessary to select the optimal type of phospholipid to achieve the optimal properties. As previously reported, the cholesterol was added during liposome preparation to enhance membrane stability and encapsulation efficiency of the payload. Additionally, the cholesterol retards the drug release which resulted in sustained release behavior, It is also reported to reduce drug leakage from liposomes in physiologic media (Farzaneh et al., 2018). 
Table (1): Optimization parameter used in the development of optimized PTX liposomal formulation

\begin{tabular}{cccc}
\hline $\begin{array}{c}\text { Optimization } \\
\text { parameters }\end{array}$ & Lipid & Drug & PTX: lipid \\
\hline Lipid type & POPC & PTX & $1: 10$ \\
& DMPC & PTX & $1: 10$ \\
& DPPC & PTX & $1: 10$ \\
PTX-to-lipid ratio & DPPC & PTX & $1: 5$ \\
& DPPC & PTX & $1: 10$ \\
& DPPC & PTX & $1: 15$ \\
& DPPC & PTX & $1: 30$ \\
& DPPC & PTX & $1: 60$ \\
\hline
\end{tabular}

As shown from figure $(2, \mathrm{~A})$, the highest PTX EE $(84.69 \pm 8.45 \%)$ was achieved for DPPC-based liposomes, followed by DMPC $(66.07 \pm 5.37 \%)$ and POPC $(48.76 \pm 6.26 \%)$. It can be noticed that a significant increase in the EE of PTX in liposomal based DPPC lipid by 1.3 -fold $(P<0.05)$ and 1.74 -fold $(P<0.01)$ when compared with DMPC and POPC based group.

The studied phospholipids have different affinity to cholesterol due to difference in fatty acid chain length, and degree of saturation. Therefore, the PTX EE may follows similar trend of affinity to the used lipids. The moderate saturated fatty acid chain phosphatidylcholine (DPPC), a saturated phosphatidylcholine with symmetric acyl chains of 16 carbons per chain, having greater affinity relative to shorter saturated phosphatidylcholine with 14 carbon acyl chain (DMPC). Additionally, the saturated fatty acid chain phosphatidylcholine possesses greater affinity relative to unsaturated chain (POPC). In summary, the EE result was in the following order: DPPC > DMPC > POPC. This result is in agreement with recent study (Schultz et al., 2019; Yuan et al., 2016). This pattern suggests that liposomes made of saturated phospholipids with longer hydrophobic tails can entrap more PTX.

Another possible reason for large differences in EE between various liposomal lipid compositions is the difference in lipid transition or melting temperature. The $T_{m}$ for DPPC equal to $41^{\circ} \mathrm{C}$, higher than room temperature, which provides better trapping of PTX in the lipid bilayer, while DMPC $\left(\mathrm{T}_{\mathrm{m}}=23{ }^{\circ} \mathrm{C}\right)$ and POPC $\left(\mathrm{T}_{\mathrm{m}}=-3{ }^{\circ} \mathrm{C}\right)$ membranes are fluid and allow for drug leakage and dissociation from the membrane.

The effects of phospholipid type on the mean diameter, PDI and ZP of liposomes were shown in figure $(2, \mathrm{~B}, \mathrm{C}, \mathrm{D})$ ). The smallest PS was achieved by DPPC based liposomes $(72.51 \pm 8.15 \mathrm{~nm})$ with the PDI of 0.0.09. Also, the DMPC-liposomes had averaged PS of $115.57 \pm 23.62 \mathrm{~nm}$ with narrow size distribution (0.24). Paradoxically, the PS of POPC showed particles diameter larger than DPPC and 
DMPC-liposomes, with an average diameter of $267.62 \pm 53.91 \mathrm{~nm}$ and PDI less than 0.4 . The polydispersity indices obtained from DLS readings are in general small which indicate that the liposomes were fairly monodispersed and that the sizes are reliable. As observed from these finding, the DPPC could produce small particle vesicles with a good homogeneity due to its physicochemical properties.

The comparatively high PS and cloudy appearance of POPC may be due to a fluid membrane character which resulted in leakage of PTX or poor affinity of PTX to the PC. Additionally, liposome nanoparticles made of POPC were sensitive to subtle changes in osmotic pressure gradients and tended to collapse and form aggregates.

Furthermore, the increase in vesicle size from DPPC to POPC could be attributed to the packing parameter and phase $\mathrm{T}_{\mathrm{m}}$. Saturated phospholipids with shorter carbohydrate chains, such as DPPC (C16:0) and DMPC (C14:0), have lower packing parameter, which is favorable for the formation of smaller particle diameters (Zhao et al., 2015). Additionally, the saturated phospholipids with shorter chain fatty acids have a relatively lower phase transition temperature, which enhanced the fluidity of bilayer and resulted in a smaller PS. Contrary, the phospholipids with monounsaturated carbohydrate chains, such as POPC (C16:0-18:1), have the highest packing parameter in addition to the low phase $\mathrm{T}_{\mathrm{m}}$ creates bigger liposomes (Jovanović et al., 2018; Zhao et al., 2015; Zhao et al., 2015). The homogeneity of the size was due to selection of lipid with favorable drug binding properties.

The ZP indicates the overall charge of a particle in the dispersion medium (Laouini et al., 2012). All the studied PC was zwitterionic types with a net neutral charge. Thus, as expected, the $\mathrm{ZP}$ of all formulation ranged from -1 to $-3 \mathrm{mV}$. It can be observed that a negligible difference in the ZP of all studied types of phospholipids. Based on the above results, the DPPC was selected for the further studied.

According to previous reports, the influence of the weight ratio of PTX to lipid and phospholipid types was considered highly vital for passive encapsulation (Rui et al., 2017). As shown in figure (3), the increase in PTX-to-lipid ratio resulted in high encapsulation efficiency of PTX. The highest encapsulation efficiency was achieved at 1: 10 (w / w) ratio of PTX to DPPC lipid, amounted to $84.69 \pm 8.45 \%$ as discussed before. Likewise, at 1: $5(\mathrm{w} / \mathrm{w})$ ratio, the encapsulation efficiency was slightly decreased but without any significant difference $(P>0.05)$. Further decrease in druglipid ratio from to 1:15 to 1: $60(\mathrm{w} / \mathrm{w})$ ratio of PTX to DPPC lipid resulted in a decrease in EE due to precipitation from 76.42 to $50.94 \%$.

These findings were confirmed by Kannan et al., (2015) who demonstrated significant increase in PTX loading by increasing PTX-to-lipid ratio (Kannan et al., 2015). The results may be due to aggregate formation that can act as nucleation sites for precipitation by decreasing in PTX-to-lipid ratio. Based on the above results, the DPPC- 
cholesterol-PTX liposomes at 1: 0.5: $0.1(\mathrm{w} / \mathrm{w})$ ratio was selected as optimized liposomal formulation to assess cellular uptake and the in vitro cytotoxicity studies.
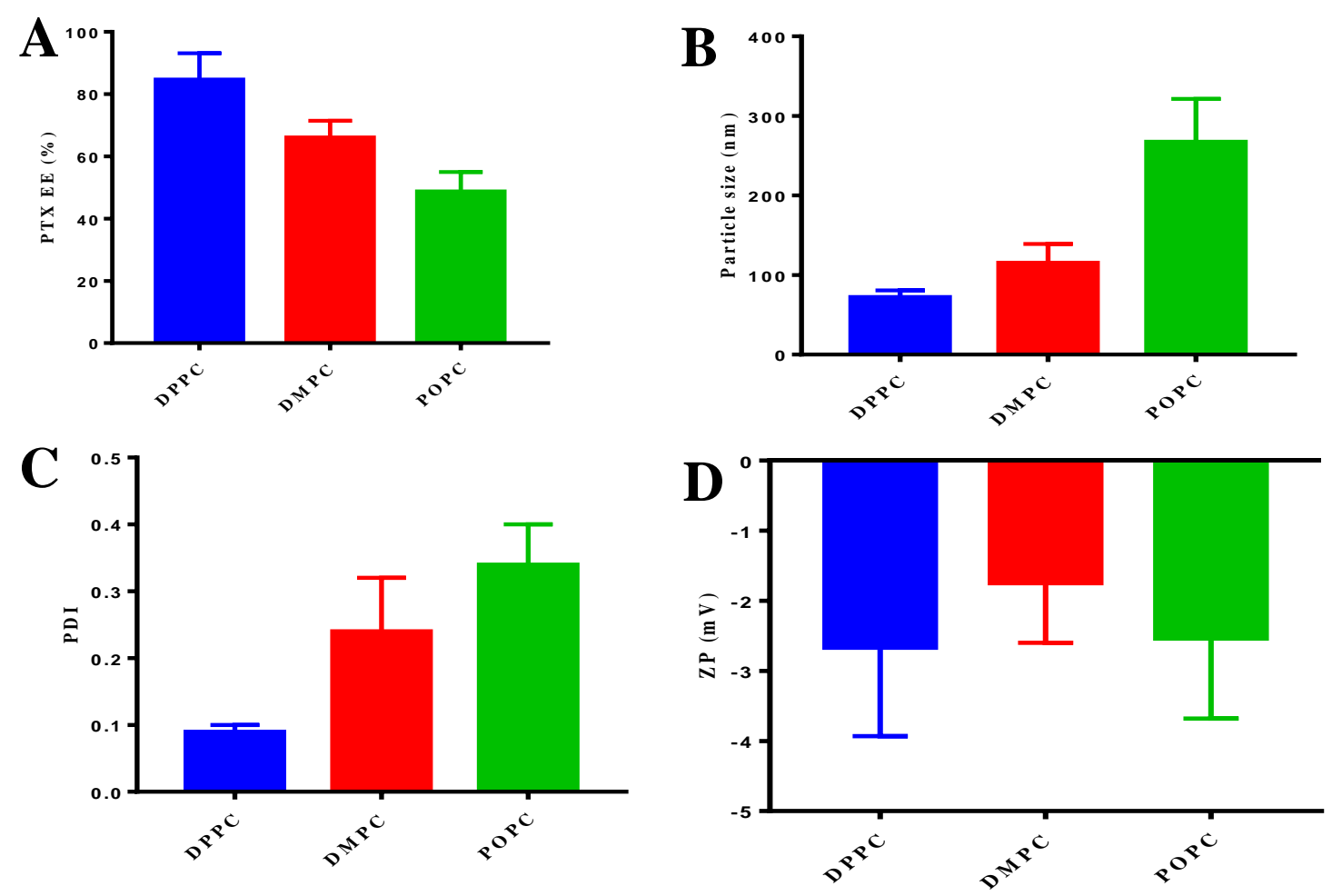

Figure (2): Effect of Effect of different zwitterionic phosphatidylcholine lipids on (A) EE, (B) PS, (C) PDI and (D) ZP of PTX-liposomal nanoparticles

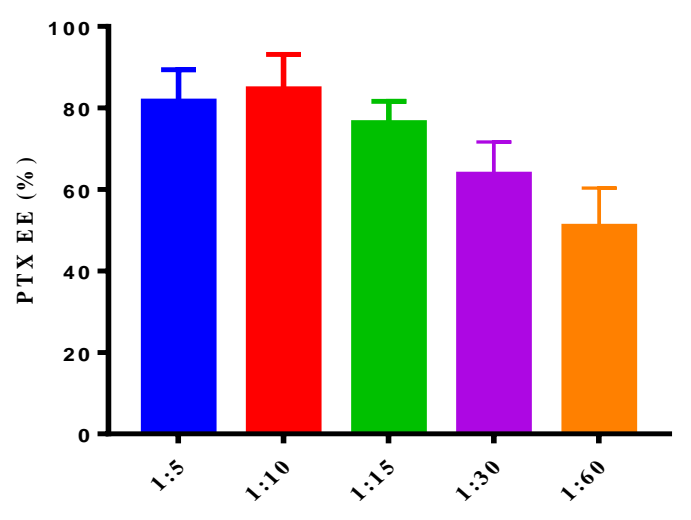

Figure (3): Effect of PTX to lipid ratio on EE at fixed ratio of cholesterol to DPPC lipid 


\section{Transmission electron microscopy}

Examination of morphology of optimized PTX loaded DPPC liposome was carried out using TEM. Figure (4) showed that the nanoparticles had a typical spherical morphology of the optimal formulation, with a uniform PS. The nanoparticles sizes showed in TEM figures were consistent with the results from DLS with an average size of $\sim 75 \mathrm{~nm}$. The image showed no coalescence between particle and no signs of drug precipitation.

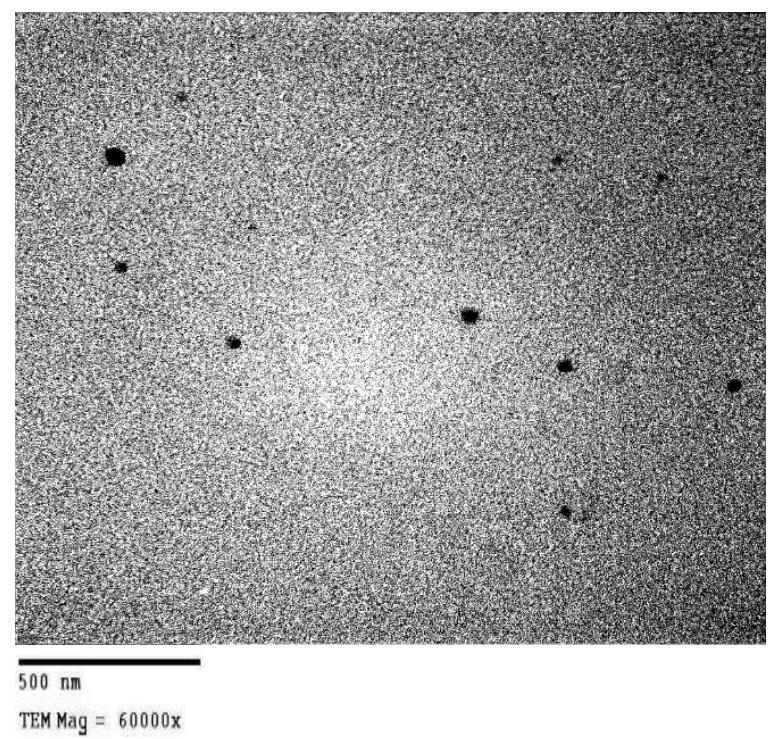

Figure (4): Transmission electron microscopy images of nanoparticles at 60,000-fold magnification. Scale bar $=500 \mathrm{~nm}$

\section{In vitro drug release study}

Dissolution studies were carried out to compare the release of PTX from optimized liposomal formulation and $\mathrm{Taxol}^{\circledR}$, and to investigate the effect of dissolution medium type on dissolution profile of PTX. The PTX release behavior PTX loaded liposome nanoparticles were investigated by dialysis at $37{ }^{\circ} \mathrm{C}$ in PBS solutions with or without $10 \% \mathrm{FBS}$, in comparison with that of drug-loaded liposomes and Taxol ${ }^{\circledR}$. Premature drug release in serum and tissue fluid may hamper the therapeutic effects of a drug and cause undesirable toxicity to normal tissues (Huang et al., 2018).

As seen in figure (5), the liposome nanoparticles demonstrated a sustained release behavior when compared to $\mathrm{Taxol}^{\circledR}{ }^{\circledR}$ (faster release); approximately $57 \%$ and $44 \%$ of PTX was cumulatively released from liposomes in the first $48 \mathrm{~h}$ under PBS / 
FBS and PBS / Tween ${ }^{\circledR} 80$, respectively, whereas the Taxol ${ }^{\circledR}$ released more than $75 \%$ and $65 \%$ of PTX after 2 days, respectively. The sustained release profile of the liposome formulation might be attributed to the presence of cholesterol, which could increase the mechanical strength of lipid membranes and reduce the drug permeability of the liposomes. It can be concluded that PTX-liposome showed significantly different release characteristics than those of the Taxol ${ }^{\circledR}$ at both studied media $(P<0.05)$.

With regard to the controlled release profile, liposome nanoparticles were considered as a favorable vehicle for the stable and safe delivery of payloads. The results suggested that PTX-liposome is significantly more stable than the Taxol ${ }^{\circledR}$ formulation and that collateral toxicity to normal tissue might be alleviated due to reduced premature release of PTX.

We assume that the PTX-liposome may circulate and be retained in the cancer cells for an extended period following administration. Considering the time required for the formulations to reach the cancer cells after administration in the body, a sustained release of the drug would be more advantageous than a rapid-releasing formulation as this is therapeutically more beneficial (Kim and Park, 2017). Therefore, PTX-liposome may be more efficient in delivering drugs via targeted drug release than is observed for Taxol $^{\circledR}$.

The results revealed that the drug was released more rapidly from Taxol ${ }^{\circledR}$ and liposomes under the blood serum situation than in PBS solutions. The comparatively slow dissolution rate in PBS may be due to the solubility of PTX in PBS with $10 \%$ FBS was higher than PBS with $0.1 \%$ Tween ${ }^{\circledR} 80$. The observations are in line with studies reported by Abouelmagd et al., (2015) who found that the dissolution rate of PTX in PBS / FBS medium was higher than PBS alone or PBS / Tween ${ }^{\circledR} 80$ (Abouelmagd et al., 2015). 

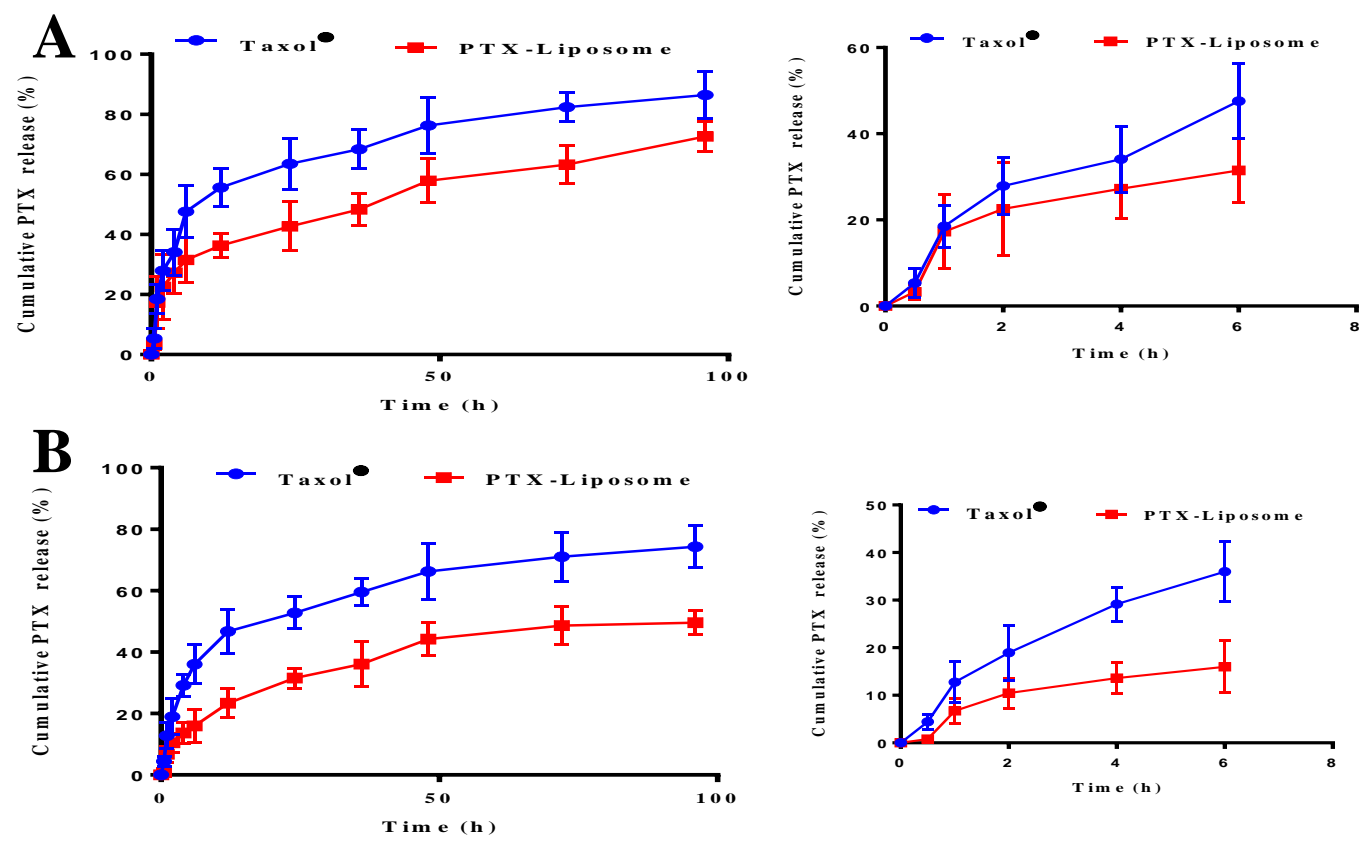

Figure (5): In vitro release profile of PTX from Taxol ${ }^{\circledR}$ and liposomes in (A) PBS with $10 \%$ FBS cell culture medium and (B) PBS with $0.1 \%$ Tween ${ }^{\circledR} 80,(\mathrm{pH} 7.4)$ at $37{ }^{\circ} \mathrm{C}$. Error bars represent the standard deviation of the mean of triplicate measurements

\section{In vitro anti-tumoral activity studies}

The in vitro cytotoxicity activity of $\mathrm{Taxol}^{\circledR}$, PTX-liposome and drug-free liposome were evaluated by the water soluble tetrazolium salt (WST-8) assay using the $\mathrm{C} 26$ cell line after $72 \mathrm{~h}$ to allow a larger number of cells to enter the G2 and M cell cycle phases during which PTX is more active. As shown in figure (6), the inhibition effects of the PTX formulations were dependent on the dose of PTX. The PTX concentration ranged from 10 to $1000 \mathrm{nM}$.

The viability of C26 cells treated with blank liposome, even at high concentrations was usually as high as $97 \%$, which indicate a good biocompatibility of the nanoparticles, for future use in drug delivery process. At the concentration of 1000 $\mathrm{nM}$ Taxol ${ }^{\circledR}$, cell viability approximately $20 \%$ was achieved after $72 \mathrm{~h}$. In case of PTXliposomes, the cell viability was slightly increased from $18.65 \%$ to $25.19 \%$ at the same concentration. Meanwhile, the $\mathrm{IC}_{50}$ was slightly increased from 150.5 to $171.5 \mathrm{nM}$, respectively, without significant difference $(P>0.05)$, which demonstrated that the PTX-liposome nanoparticles developed a foreseeable effect in vitro anti-tumor efficacy. The $\mathrm{IC}_{50}$ of studied groups were illustrated in table (2).

It should be emphasized that in the case of Taxol $^{\circledR}$ a significant effect was attributed to not only PTX, but also the excipient Cremophor ${ }^{\circledR}$ EL (especially at higher concentration), whereas in the case of PTX-loaded liposomes nanoparticles, the 
cytotoxicity observed was only attributed to PTX (drug-free nanoparticles were noncytotoxic). only. This decrease in cell viability, measured by the CCK test can resulted from an inhibition of cell growth or from cytotoxicity. The slightly increase in cell viability and IC50 of liposomes may also attributed to the sustained release effect of liposome nanoparticles. The physicochemical properties of PTX-liposomes make it an ideal transporter of anticancer agents.

Table (2): The half maximal inhibitory concentration data of Taxol ${ }^{\circledR}$ and PTXliposomes against $\mathrm{C} 26$ cancer cell line after $72 \mathrm{~h}$ incubation

\begin{tabular}{ccc}
\hline Parameters & Taxol $^{\circledR *}$ & PTX-liposome $^{*}$ \\
\hline $\mathrm{R}^{2}$ & 0.95 & 0.992 \\
$\operatorname{Log~IC}$ & $2.23 \pm 0.07$ & $2.177 \pm 0.08$ \\
$\mathrm{IC}_{50}(\mathrm{nM})$ & $150.5 \pm 13.8$ & $171.5 \pm 32.11$ \\
\hline
\end{tabular}

*Data expressed as a mean $\pm \mathrm{SD},(\mathrm{n}=5)$

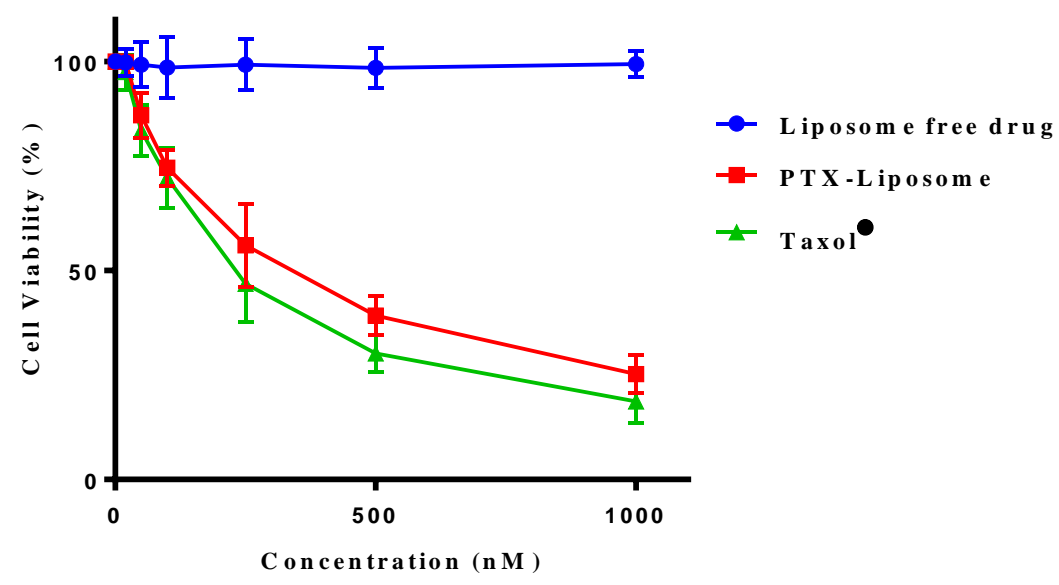

Figure (6): Survival rates of C26 tumor cells exposing to the liposomes with or without paclitaxel and Taxol ${ }^{\circledR}$. The amount of the liposomes corresponding to the paclitaxel liposome was added as the control. The cytotoxicity was measured by CCK- 8 assay. Data are presented as the mean \pm standard deviation, $(n=5)$

\section{Conclusion}

The type of phospholipid was found to play a critical role in the stabilization of liposomal membranes and affected various features of liposomal PTX, including PS distribution, PTX EE and PTX leakage kinetics in different media. The DPPC formulation exhibited supremacy in all physicochemical characteristics compared with DMPC and POPC formulations and illustrated a sustained release behavior when 
compared with Taxol ${ }^{\circledR}$, which is an essential characteristic to decrease the systemic toxicity in vivo. Thus, the DPPC-liposomes loaded with PTX formulation can be considered as an alternative to the marketed drug product. Accordingly, the PTX encapsulated DDPC liposomes would be considered as a promising drug formulation delivery of PTX and management colorectal cancer cells.

\section{REFERENCES}

Abouelmagd, S.A., Sun, B., Chang, A.C., Ku, Y.J., Yeo, Y., (2015). Release kinetics study of poorly water-soluble drugs from nanoparticles: Are we doing it right? Mol. Pharm. 12, 997-1003. https://doi.org/10.1021/mp500817h

Alves, A.C., Magarkar, A., Horta, M., Lima, J.L.F.C., Bunker, A., Nunes, C., Reis, S., (2017). Influence of doxorubicin on model cell membrane properties: insights from in vitro and in silico studies. Sci. Rep. 7, 1-11.

Davis, M.E., Chen, Z.G., Shin, D.M., (2008). Nanoparticle therapeutics : an emerging treatment modality for cancer 7, 771-782. https://doi.org/10.1038/nrd2614

Ding, D., Sun, B., Cui, W., Chen, Q., Zhang, X., (2019). Integration of phospholipiddrug complex into self-nanoemulsifying drug delivery system to facilitate oral delivery of paclitaxel 14, 552-558. https://doi.org/10.1016/j.ajps.2018.10.003

Farzaneh, H., Ebrahimi Nik, M., Mashreghi, M., Saberi, Z., Jaafari, M.R., Teymouri, M., (2018). A study on the role of cholesterol and phosphatidylcholine in various features of liposomal doxorubicin: From liposomal preparation to therapy. Int. J. Pharm. 551, 300-308. https://doi.org/10.1016/j.ijpharm.2018.09.047

Huang, S.T., Wang, Y.P., Chen, Y.H., Lin, C.T., Li, W.S., Wu, H.C., (2018). Liposomal paclitaxel induces fewer hematopoietic and cardiovascular complications than bioequivalent doses of Taxol. Int. J. Oncol. 53, 1105-1117. https://doi.org/10.3892/ijo.2018.4449

Jovanović, A.A., Balanč, B.D., Ota, A., Ahlin Grabnar, P., Djordjević, V.B., Šavikin, K.P., Bugarski, B.M., Nedović, V.A., Poklar Ulrih, N., (2018). Comparative Effects of Cholesterol and $\beta$-Sitosterol on the Liposome Membrane Characteristics. Eur. J. Lipid Sci. Technol. 120, 1-11. https://doi.org/10.1002/ejlt.201800039

Kannan, V., Balabathula, P., Divi, M.K., Thoma, L.A., Wood, G.C., (2015). Optimization of drug loading to improve physical stability of paclitaxel-loaded long-circulating liposomes. J. Liposome Res. 25, 308-315. https://doi.org/10.3109/08982104.2014.995671

Kim, H., Nobeyama, T., Honda, S., Yasuda, K., Morone, N., Murakami, T., (2019). Membrane fusogenic high-density lipoprotein nanoparticles. Biochim. 
Biophys. Acta $\quad$ - $\quad$ Biomembr. $1861, \quad 18008$. https://doi.org/10.1016/j.bbamem.2019.06.007

Kim, J.E., Park, Y.J., (2017). Paclitaxel-loaded hyaluronan solid nanoemulsions for enhanced treatment efficacy in ovarian cancer. Int. J. Nanomedicine 12, 645658. https://doi.org/10.2147/IJN.S124158

Laouini, A., Jaafar-Maalej, C., Limayem-Blouza, I., Sfar, S., Charcosset, C., Fessi, H., (2012). Preparation, Characterization and Applications of Liposomes: State of the Art. J. Colloid Sci. Biotechnol. 1, 147-168. https://doi.org/10.1166/jcsb.2012.1020

Luo, W., Xu, X., Zhou, B., He, P., Li, Y., Liu, C., (2019). Materials Science \& Engineering C Formation of enzymatic / redox-switching nanogates on mesoporous silica nanoparticles for anticancer drug delivery. Mater. Sci. Eng. C 100, 855-861. https://doi.org/10.1016/j.msec.2019.03.028

Nordin, N., Yeap, S.K., Rahman, H.S., Zamberi, N.R., (2019). In vitro cytotoxicity and anticancer effects of citral nanostructured lipid carrier on MDA MBA-231 human breast cancer cells. Sci. Rep. 1-19. https://doi.org/10.1038/s41598-01838214-x

Ohvo-Rekilä, H., Mattjus, P., Slotte, J.P., (1998). The influence of hydrophobic mismatch on androsterol/phosphatidylcholine interactions in model membranes. Biochim. Biophys. Acta (BBA)-Biomembranes 1372, 331-338.

Rui, M., Xin, Y., Li, R., Ge, Y., Feng, C., Xu, X., (2017). Targeted Biomimetic Nanoparticles for Synergistic Combination Chemotherapy of Paclitaxel and Doxorubicin. Mol. Pharm. 14, 107-123. https://doi.org/10.1021/acs.molpharmaceut.6b00732

Sakai-Kato, K., Sakurai, M., Takechi-Haraya, Y., Nanjo, K., Goda, Y., (2017). Involvement of scavenger receptor class B type 1 and low-density lipoprotein receptor in the internalization of liposomes into HepG2 cells. Biochim. Biophys. Acta - Biomembr. 1859, 2253-2258. https://doi.org/10.1016/j.bbamem.2017.09.005

Schultz, M.L., Fawaz, M. V, Azaria, R.D., Hollon, T.C., Liu, E.A., Kunkel, T.J., Halseth, T.A., Krus, K.L., Ming, R., Morin, E.E., Mcloughlin, H.S., Bushart, D.D., Paulson, H.L., Shakkottai, V.G., Orringer, D.A., Schwendeman, A.S., Lieberman, A.P., (2019). Synthetic high-density lipoprotein nanoparticles for the treatment of Niemann - Pick diseases 1-18.

Tang, J., Kuai, R., Yuan, W., Drake, L., Moon, J.J., Schwendeman, A., (2017). Effect of size and pegylation of liposomes and peptide-based synthetic lipoproteins on tumor targeting. Nanomedicine Nanotechnology, Biol. Med. 13, 1869-1878. https://doi.org/10.1016/j.nano.2017.04.009 
Villar, A.M.S., Naveros, B.C., Campmany, A.C.C., Trenchs, M.A., Rocabert, C.B., Bellowa, L.H., (2012). Design and optimization of self-nanoemulsifying drug delivery systems (SNEDDS) for enhanced dissolution of gemfibrozil. Int. J. Pharm. 431, 161-175. https://doi.org/10.1016/j.ijpharm.2012.04.001

Yuan, Y., Wen, J., Tang, J., Kan, Q., Ackermann, R., Olsen, K., Schwendeman, A., (2016). Synthetic high-density lipoproteins for delivery of 10hydroxycamptothecin. Int. J. Nanomedicine 11, 629-6238. https://doi.org/10.2147/IJN.S112835

Zhao, L., Temelli, F., Curtis, J.M., Chen, L., (2015). Preparation of liposomes using supercritical carbon dioxide technology: Effects of phospholipids and sterols. Food Res. Int. 77, 63-72. https://doi.org/10.1016/j.foodres.2015.07.006

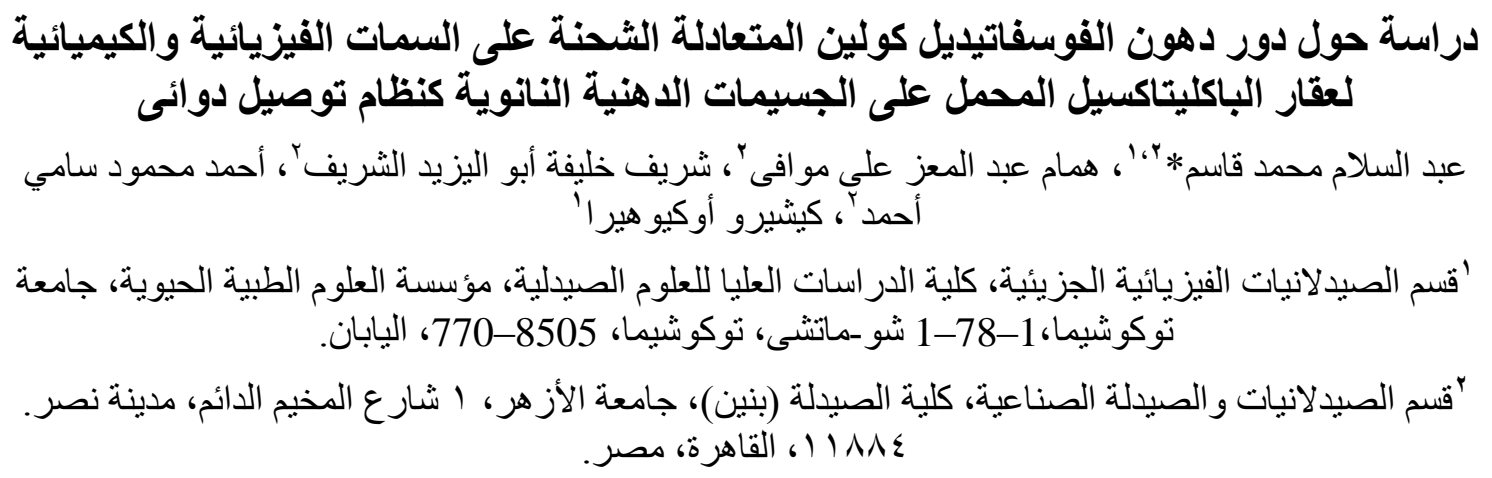

dr.abdokasem2011@azhar.edu.eg : البريد الاكتروني للباحث الرئيسي *

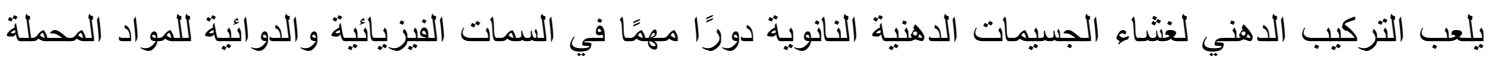

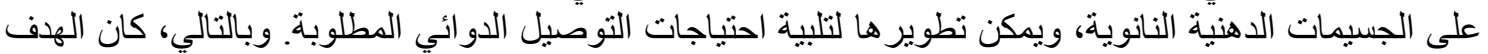

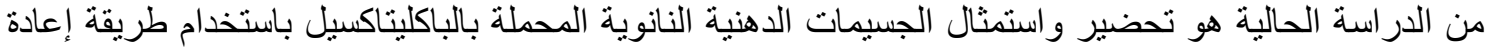

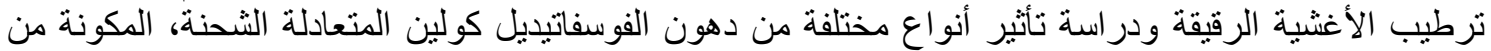

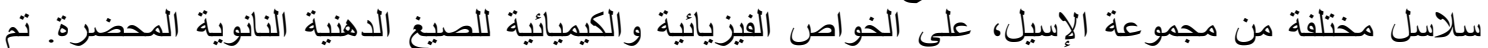

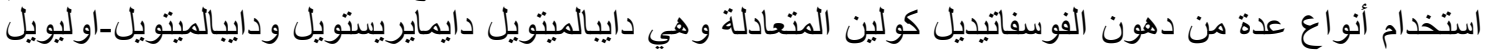

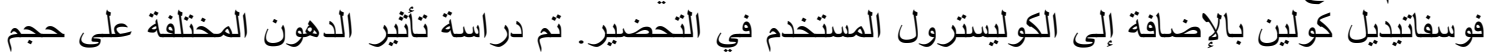

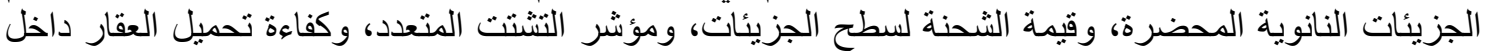

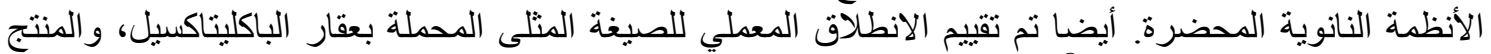

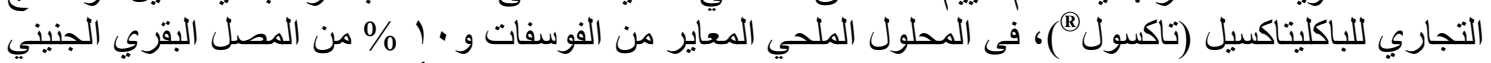

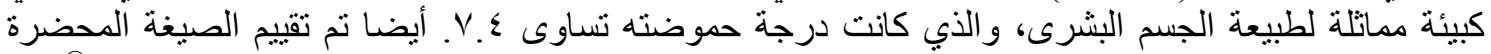

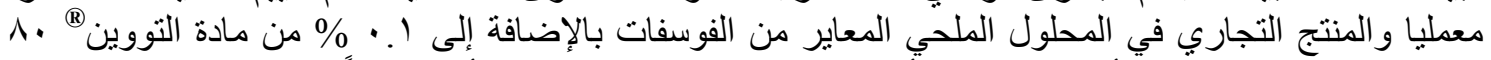

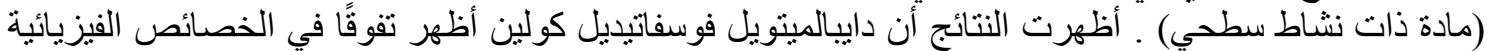

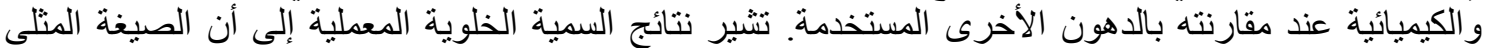

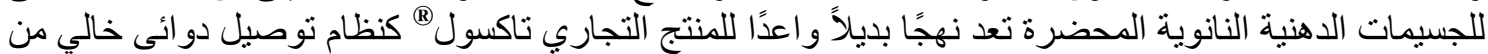

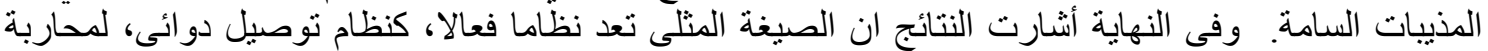

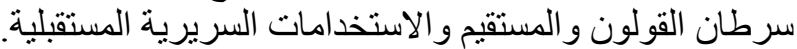
الكلمات المفتاحية : الجسيمات الدهنية النانوية، باكتسيل، كوليستيرول، دهون، فوسفاتيديل كولين، الانطلاق المعملي المعدل 